\title{
Incremental costs of fragility fractures: a population-based matched -cohort study from Ontario, Canada
}

\author{
J.-É. Tarride ${ }^{1,2,3}$ • J. D. Adachi ${ }^{4}$ J. P. Brown ${ }^{5} \cdot$ E. Schemitsch ${ }^{6} \cdot$ L. Slatkovska $^{7}$ - N. Burke ${ }^{7}$
}

Received: 15 October 2020 / Accepted: 4 February 2021 / Published online: 18 February 2021

(C) The Author(s) 2021

\begin{abstract}
Summary Using a matched cohort design, the 1-year excess cost of incident fragility fractures at any site was $\$ 26,341$ per patient, with $43 \%$ of total excess costs attributed to hospitalization. The high economic burden of fractures in Ontario underscores the urgency of closing the secondary fracture prevention gap.

Introduction This retrospective real-world observational study was conducted to document the incremental costs associated with fragility fractures in Ontario, Canada.

Methods Patients aged $>65$ years with an index fragility fracture occurring between January 2011 and March 2015 were identified from administrative databases and matched 1:1 to a cohort of similar patients without a fracture. Healthcare resource utilization data were extracted from healthcare records and associated costs were calculated on a per-patient level and for the province of Ontario. Costs were presented as 2017 Canadian dollars.

Results The eligible cohort included 115,776 patients with a fragility fracture. Of these, 101,773 patients were successfully matched 1:1 to a non-fracture cohort. Overall, hip fractures $(n=31,613)$ were the most common, whereas femur fractures $(n=$ 3002 ) were the least common type. Hospitalization and continuing care/home care/long-term care accounted for more than $60 \%$ of 1-year direct costs, whereas $5 \%$ was attributed to medication costs. First-year costs per patient in the fracture cohort were approximately threefold higher versus the non-fracture cohort (mean $\$ 37,362$ versus $\$ 11,020$, respectively). The incremental first-year direct healthcare costs of fragility fractures for the province of Ontario were calculated at $\$ 724$ million per year.

Conclusions Fragility fractures were associated with a threefold increase in overall mean healthcare costs per patient compared to patients without fractures. With an aging population, there is an urgent need for improved prevention strategies for patients at high-risk of fracture to decrease the economic burden of fragility fractures on the Canadian healthcare system.
\end{abstract}

Keywords Cost of illness $\cdot$ Fragility fracture $\cdot$ Healthcare resource utilization $\cdot$ Matched cohort $\cdot$ Osteoporosis

N. Burke

nburke02@amgen.com

1 Department of Health Research Methods, Evidence and Impact (HEI), McMaster University, Hamilton, Ontario, Canada

2 Programs for Assessment of Technology in Health, The Research Institute of St. Joe's Hamilton, St Joseph's Healthcare Hamilton, Hamilton, Ontario, Canada

3 Centre for Health Economics and Policy Analysis (CHEPA), McMaster University, Hamilton, Ontario, Canada

4 Department of Medicine, McMaster University, Hamilton, Ontario, Canada

5 Department of Medicine, Division of Rheumatology, CHU de Québec Research Centre, Laval University, Québec City, Québec, Canada

6 Department of Surgery, Western University, London, Ontario, Canada

7 Amgen Canada Inc, Mississauga, Ontario, Canada

\section{Introduction}

Due to its increasing prevalence and associated costs, fragility fractures are a major public health issue. Worldwide, the number of individuals with fractures due to osteoporosis has increased from 56.2 million to 157.4 million between 2000 and 2010 and this number is expected to increase at least twofold until 2040 [1,2]. Despite variations in the incidence rates of individual fracture types across countries, the pattern of fractures appears to be similar in all developed countries examined, with major osteoporotic fractures comprising the majority of all recorded incidents [3]. Fragility fractures impose a substantial economic burden, estimated at $€ 37.5$ billion in the largest 5 countries of the European Union plus Sweden (EU6) in 2017 and $\$ 19$ billion in the USA in 2005 (equivalent to $\$ 42$ billion and \$26 billion respectively, in 2020 USD values) [4, 
5]. Cost of illness studies comparing patients with and without fractures have been conducted using claims data from the USA [6, 7], and healthcare records from the UK $[8,9]$, the Netherlands [10], and Belgium [11]. These studies have demonstrated that treatment and care of patients with fractures imposes a substantial burden on healthcare systems compared to patients of a similar age and sex without fractures, emphasizing the importance of primary and secondary prevention in high-risk individuals.

However, these studies were not Canadian and are not useful to support economic evaluations of osteoporosis in Canada [12]. While several studies have documented the costs associated with osteoporosis in Canada [13-16], very few recent studies have evaluated the incremental costs associated with fragility fractures. One of the first studies, using 1995-2008 administrative data from the province of Manitoba (4\% of Canadian population), estimated the incremental costs associated with fractures, with hip fracture having the highest incremental cost over a 2-year period of approximately $\$ 45,000$ per patient [14]. A more recent study using data from Ontario ( $40 \%$ of Canadian population) reported that the first-year incremental direct healthcare costs associated with hip fracture were $\$ 282$ million in Ontario or $\$ 1.1$ billion in Canada in 2010 [15]. However, this study was limited to hip fractures and the current incremental burden associated with all types of fragility fractures in Canada is unknown. To better inform policy makers, payers, clinicians, and patients, the primary objective of this study was to document the incremental costs associated with fractures.

\section{Methods}

This was a retrospective population-based observational study using de-identified health services data of patients in the province of Ontario (population 14.7 million) from the ICES Data Repository [17].

\section{Fracture cohort}

Fractures were identified using International Classification of Disease (ICD)-10-CA diagnostic codes for the following osteoporotic fracture sites: hip, femur, vertebral (clinical), wrist (distal radius, or both distal radius and ulna), clavicle/ribs/ sternum, humerus, tibia/fibula/knee (including medial and lateral malleolus), pelvis, radius/ulna (proximal, midshaft, or distal ulna only), and multisite (Online Resource Table 1). Fractures were captured from hospital admissions or ambulatory care visits where fracture was the "most responsible diagnosis" or "pre-admit comorbidity" (from Discharge Abstract Database (DAD) and Same Day Surgery (SDS) databases) or if fracture was the "most responsible diagnosis" or a "coexisting condition" at the time of an emergency room visit (from National Ambulatory Care Reporting System (NACRS) database). Patients were included in the cohort if they were 66 years or older $(>65)$ and had an index fragility fracture between January 1, 2011, and March 31, 2015 and were followed until March 31, 2017 (Online Resource Figure 1). Therefore, the opportunity for follow-up ranged from 2 years (2015-2017) to 6 years (2011-2017). There was a look back period of 5 years from the index date for prior events. Patients were limited to adults aged $>65$ in order to analyze medication data based on public drug coverage under the Ontario Drug Benefit (ODB) Program for at least 1 year prior to the index fracture. To maximize the probability that only fragility fractures were examined, patients were excluded if they had fractures at non-osteoporotic fracture sites (i.e., skull, face, hands, and feet) or fractures associated with a trauma code (Online Resource Table 1). Patients were also excluded if they experienced a fragility fracture within 5 years prior to the index date to minimize the likelihood that examined outcomes were related to a recent fracture occurring prior to an index event.

\section{Non-fracture cohort}

Patients with fractures were matched 1:1 to persons without a fracture (non-fracture cohort). Eligible controls included individuals in the Ontario Registered Persons Database who did not have a fracture during the study period (2011-2015) or during the 5-year lookback period. The two cohorts were hard matched by month and year of index date (a random index date was assigned to controls prior to matching based on the distribution of the index date among cases) according to the following a priori specified characteristics: sex, age category (66-70, 71-75, 76-80, 81-85, $\geq 86$ years), geography (urban/ rural), and comorbidities. ICES-derived cohorts with validated case-finding algorithms were available for some comorbidities (i.e., asthma, chronic obstructive pulmonary disease (COPD), rheumatoid arthritis, diabetes, myocardial infraction, dementia) but not for others (i.e., psoriasis, spondyloarthritis, cancer, chronic kidney disease, stroke, osteoarthritis). Comorbidities were captured at any time prior to the index date, except cancer which was captured within 5 years. For every case, a single matched control was selected using a greedy matching algorithm [18] which finds the best match among the currently available choices and once a match is made, that match is not reconsidered.

\section{Healthcare resource costs}

The costs associated with the following types of healthcare resource utilization were included in the study: hospitalizations (i.e., inpatient hospitalization, same day surgery), inpatient rehabilitation, continuing care services (i.e., hospitalbased continuing care (e.g., chronic or complex care beds), 
home care, long-term care (LTC)), prescription drug benefit claims, and other healthcare services (i.e., emergency department visits, hospital outpatient clinic visits, physician billings, non-physician billings, laboratory claims). Billings for nonphysicians included physiotherapy services that are covered under the Ontario Health Insurance Plan (OHIP). Costs of fracture-related surgical procedures (initial or revision) included the cost of prostheses. The primary healthcare services datasets used for this study are described in Online Resource Table 2. The cost data was reported in the year that they occurred and standardized to 2017 Canadian dollar values. The healthcare payer perspective was taken in this study and only direct costs were considered.

Direct healthcare resource utilization costs were calculated and described using a previously published algorithm [19]. A bottom-up or micro-level costing approach was taken, which starts with individual encounters or utilization in the health system and attached prices (or costs or amounts paid) to each encounter. Two primary components were required to measure patient-level healthcare expenditures: utilization data (such as length of stay, intensity of resource usage, readmission) and cost information (such as cost per stay or per day). Person-level costs were derived by linking the utilization data from administrative healthcare databases to both individual provider and corporate cost information collected by the Ontario Ministry of Health and Long-Term Care. Data for patient-level costs was aggregated to estimate the direct cost of publicly paid healthcare services for the population of interest and over the time period of this study. All individuals had at least 1 year of follow-up, so everyone contributed costs for year 1, but not necessarily for year 2 and beyond.

\section{Data synthesis and analysis}

Descriptive statistics (mean and standard deviation [SD], medians, and interquartile ranges [IQR]) were used to summarize baseline characteristics of the patient population as well as outcomes. For the primary analysis, annual healthcare costs attributable to fractures were calculated by comparing total and service-specific direct healthcare costs of the fracture cohort to the matched non-fracture cohort in the first year after a fragility fracture. The results were first presented on a perpatient basis and for the province of Ontario. These results were extrapolated to Canada based on the ratio of the Canadian (37.9 million) and Ontario (14.7 million) populations (i.e., 2.6). To assess uncertainty in the extrapolation of the results to the national level due to potential differences in unit costs or population distribution over 65 years and older, a sensitivity analysis was conducted by increasing and decreasing the per-patient excess costs observed in Ontario by $10 \%$ when extrapolating the results to Canada. In secondary analyses, 1-year fracture cost data were described based on gender, age, number of comorbidities, site of fracture, and by year of index fracture (2011-2015). Cases were captured up to March 31, 2015 but were projected for the entire calendar year by multiplying by a factor of 4 . Although the first-year cost for fractures was used to provide baseline assessment of the economic burden against those without fractures, we also documented for our fracture cohort healthcare costs per patient up to 5 years following an index fracture. All analyses were performed by ICES Data and Analytic Services and provided as aggregate-level results.

\section{Results}

\section{Patient demographics}

A total of 115,776 patients aged $>65$ were identified in Ontario with an index fragility fracture in the study period and comprised the full fracture cohort. Approximately $88 \%$ of cases $(N=101,773)$ were successfully matched to an equal number of non-fracture controls and comprised the matched fracture cohort. Details of the patients included in the analysis are described in Online Resource Figure 2. The baseline characteristics of patients in these cohorts are described in Table 1. The 2 cohorts were matched by sex ( $72 \%$ female), age (mean 80.4 years), and comorbidities captured from the healthcare records ( $76.2 \%$ of patients with comorbid osteoarthritis, $40 \%$ with asthma or COPD, and a similar proportion with a stroke or a vascular event in each cohort). In the full fracture cohort, $28.3 \%$ received osteoporosis treatment within 1 year of the index date. Osteoporosis treatments, which were not used for matching, were dispensed in $20.8 \%$ of the non-fracture cohort. The majority (70\%) of fractures were treated in large community hospitals.

\section{Fracture types and first-year healthcare costs}

The most common sites of index fracture were the hip ( $n=$ $31,613)$, wrist $(n=17,859)$, and the clavicle, ribs, and sternum $(n=14,559)$. The least common sites were the radius and ulna $(n=4828)$ and the femur $(n=3002)$. Multiple site fractures $(n$ $=3735$ ) included a combination of 7340 fracture codes (mean $2.05 \pm$ SD 0.23 , minimum of 2 and maximum of 5) of which hip $(n=1260)$, wrist $(n=1190)$, humerus $(n=1181)$, and pelvis $(n=1002)$ accounted for almost two-thirds of all the combination codes. The number of incident fracture cases increased steadily from $2011(n=25,154)$ to 2015 ( $n=$ 28,892), and across each fracture site as described in Online Resource Figure 3.

The mean \pm SD annual healthcare cost per patient for all index fracture sites in the first-year post-index fracture was $\$ 39,089 \pm \$ 43,272$ (Fig. 1). Femur fractures were the least common fracture site; however, they were associated with the highest mean cost per patient at $\$ 65,490 \pm \$ 54,116$. 
Table 1 Characteristics of fracture and non-fracture cohorts, Ontario, 2011 to 2015

\begin{tabular}{|c|c|c|c|}
\hline Clinical characteristics & $\begin{array}{l}\text { Full fracture cohort } \\
n(\%)\end{array}$ & $\begin{array}{l}\text { Matched fracture cohort } \\
n(\%)\end{array}$ & $\begin{array}{l}\text { Matched non-fracture cohort } \\
n(\%)\end{array}$ \\
\hline Total number of patients & 115,776 & 101,773 & 101,773 \\
\hline \multicolumn{4}{|l|}{ Sex } \\
\hline Female & $83,690(72.3 \%)$ & $74,557(73.3 \%)$ & $74,557(73.3 \%)$ \\
\hline Male & $32,086(27.7 \%)$ & $27,216(26.7 \%)$ & $27,216(26.7 \%)$ \\
\hline \multicolumn{4}{|l|}{ Age } \\
\hline Mean $\pm \mathrm{SD}^{\mathrm{a}}$ & $80.41 \pm 8.28$ & $80.25 \pm 8.37^{*}$ & $80.33 \pm 8.75 *$ \\
\hline Median $(\mathrm{IQR})^{\mathrm{a}}$ & $81(74-87)$ & $81(73-87)$ & $81(73-87)$ \\
\hline 66--70 years & $17,998(15.5 \%)$ & $16,672(16.4 \%)$ & $16,672(16.4 \%)$ \\
\hline $71-75$ years & $17,847(15.4 \%)$ & $15,996(15.7 \%)$ & $15,996(15.7 \%)$ \\
\hline $76-80$ years & $20,596(17.8 \%)$ & $17,952(17.6 \%)$ & $17,952(17.6 \%)$ \\
\hline $81-85$ years & $24,119(20.8 \%)$ & $20,584(20.2 \%)$ & $20,584(20.2 \%)$ \\
\hline$\geq 86$ years & $35,216(30.4 \%)$ & $30,569(30.0 \%)$ & $30,569(30.0 \%)$ \\
\hline \multicolumn{4}{|l|}{ Respiratory conditions $^{\mathrm{b}}$} \\
\hline Asthma & $17,538(15.1 \%)$ & $13,113(12.9 \%)$ & $13,113(12.9 \%)$ \\
\hline COPD & $33,485(28.9 \%)$ & $25,991(25.5 \%)$ & $25,991(25.5 \%)$ \\
\hline \multicolumn{4}{|l|}{ Inflammatory conditions ${ }^{\mathrm{b}}$} \\
\hline Rheumatoid arthritis & $4459(3.9 \%)$ & $2208(2.2 \%)$ & $2208(2.2 \%)$ \\
\hline Psoriasis & $8076(7.0 \%)$ & $4985(4.9 \%)$ & $4985(4.9 \%)$ \\
\hline Spondyloarthritis & $5084(4.4 \%)$ & $2432(2.4 \%)$ & $2432(2.4 \%)$ \\
\hline Cancer $^{\mathrm{b}}$ & $8390(7.2 \%)$ & $5166(5.1 \%)$ & $5166(5.1 \%)$ \\
\hline Chronic kidney disease $^{\mathrm{b}}$ & $13,757(11.9 \%)$ & $8909(8.8 \%)$ & $8909(8.8 \%)$ \\
\hline Diabetes $^{\mathrm{b}}$ & $35,434(30.6 \%)$ & $29,074(28.6 \%)$ & $29,074(28.6 \%)$ \\
\hline \multicolumn{4}{|l|}{ Vascular events $^{\mathrm{b}}$} \\
\hline Myocardial infarction & $8175(7.1 \%)$ & $4549(4.5 \%)$ & $4549(4.5 \%)$ \\
\hline Stroke or cerebrovascular event & $35,030(30.3 \%)$ & $28,015(27.5 \%)$ & $28,015(27.5 \%)$ \\
\hline Osteoarthritis $^{\mathrm{b}}$ & $88,223(76.2 \%)$ & $77,526(76.2 \%)$ & $77,526(76.2 \%)$ \\
\hline Dementia $^{\mathrm{b}}$ & $24,092(20.8 \%)$ & $18,359(18.0 \%)$ & $18,359(18.0 \%)$ \\
\hline \multicolumn{4}{|c|}{ Osteoporosis treatment type within 1 year prior $^{\mathrm{a}, \mathrm{c}}$} \\
\hline Any treatment & $32,757(28.3 \%)$ & $28,974(28.5 \%)^{* *}$ & $21,179(20.8 \%)^{* *}$ \\
\hline Denosumab & $1578(1.4 \%)$ & $1383(1.4 \%)^{* *}$ & $1088(1.1 \%)^{* *}$ \\
\hline Bisphosphonate & $29,030(25.1 \%)$ & $25,626(25.2 \%)^{* *}$ & $17,720(17.4 \%)^{* *}$ \\
\hline Raloxifene & $656(0.6 \%)$ & $599(0.6 \%)^{* *}$ & $465(0.5 \%) * *$ \\
\hline HRT & $3597(3.1 \%)$ & $3259(3.2 \%)$ & $3312(3.3 \%)$ \\
\hline \multicolumn{4}{|l|}{ Index fracture by site ${ }^{\mathrm{a}, \mathrm{d}}$} \\
\hline Hip & $31,613(27.3 \%)$ & $26,963(26.5 \%)$ & - \\
\hline Wrist & $17,859(15.4 \%)$ & $16,467(16.2 \%)$ & - \\
\hline Clavicle/ribs/sternum & $14,559(12.6 \%)$ & $12,630(12.4 \%)$ & - \\
\hline Humerus & $13,237(11.4 \%)$ & $11,756(11.6 \%)$ & - \\
\hline Tibia/fibula/knee & $10,894(9.4 \%)$ & $9859(9.7 \%)$ & - \\
\hline Pelvis & $8328(7.2 \%)$ & $7209(7.1 \%)$ & - \\
\hline Vertebral & $7721(6.7 \%)$ & $6595(6.5 \%)$ & - \\
\hline Radius/ulna & $4828(4.2 \%)$ & $4377(4.3 \%)$ & - \\
\hline Multisite & $3735(3.2 \%)$ & $3299(3.2 \%)$ & - \\
\hline Femur & $3002(2.6 \%)$ & $2618(2.6 \%)$ & - \\
\hline \multicolumn{4}{|l|}{ Fragility fracture treatment location ${ }^{\mathrm{a}}$} \\
\hline Urban & $103,720(89.6 \%)$ & - & - \\
\hline Rural & $10,626(9.2 \%)$ & - & - \\
\hline Missing & $1430(1.2 \%)$ & - & - \\
\hline
\end{tabular}


Table 1 (continued)

\begin{tabular}{llll}
\hline Clinical characteristics & $\begin{array}{l}\text { Full fracture cohort } \\
n(\%)\end{array}$ & $\begin{array}{l}\text { Matched fracture cohort } \\
n(\%)\end{array}$ & $\begin{array}{l}\text { Matched non-fracture cohort } \\
n(\%)\end{array}$ \\
\hline $\begin{array}{l}\text { Fragility fracture treatment location type } \\
\text { Large community }\end{array}$ & $80,638(69.7 \%)$ & & - \\
Small community & $6324(5.5 \%)$ & - & - \\
Teaching & $27,380(23.6 \%)$ & - & - \\
Missing & $1434(1.2 \%)$ & - & - \\
\hline
\end{tabular}

Values reported as $n(\%)$ unless otherwise indicated, percent of total respective cohort

${ }^{*} p<0.05, * * p<0.001$ statistical significance between fracture cohort and non-fracture cohort

${ }^{a}$ Variable not used for matching

${ }^{\mathrm{b}}$ Time frame for cancer was 5 years within index date and for all other comorbidities any time prior to index date

${ }^{\mathrm{c}}$ Dispensed within 1 year of index date. Bisphosphonates include alendronate, etidronate, risedronate, or zoledronic acid

${ }^{\mathrm{d}}$ Index fragility fracture cases from January 1, 2011 to March 31, 2015

$C O P D$ chronic obstructive pulmonary disease, $H R T$ hormone replacement therapy, $I Q R$ interquartile range, $S D$ standard deviation

Wrist fractures incurred the lowest mean cost per patient at $\$ 16,541 \pm \$ 25,687$. The proportion of different types of costs for all index fracture sites was highest for hospitalizations (37\%), followed by continuing care/home care/LTC (31\%). The lowest proportion of costs were attributed to prescription drugs $(5 \%)$. Although there were variations in costs between index fracture sites, similar trends were observed for most fracture sites, e.g., hospitalization and continuing care accounted for the largest portion of direct costs. However, fractures of the upper limb (wrist and radius/ulna) and pelvis had substantial proportion of costs for "other healthcare services" associated with outpatient care of these fractures (e.g., hospital outpatient clinic visits, non-physician billings such as physiotherapists). Mean cost per patient in the first year following a fracture increased with age (Online Resource Figure 4) and number of comorbidities across all index fracture sites (Online Resource Figure 5).

\section{First-year incremental costs associated with fragility fractures}

In the matched cohort of patients without a fragility fracture, the mean $\pm \mathrm{SD}$ total healthcare costs in the first year after index date were $\$ 11,020 \pm \$ 21,436$ (Fig. 2), with the highest

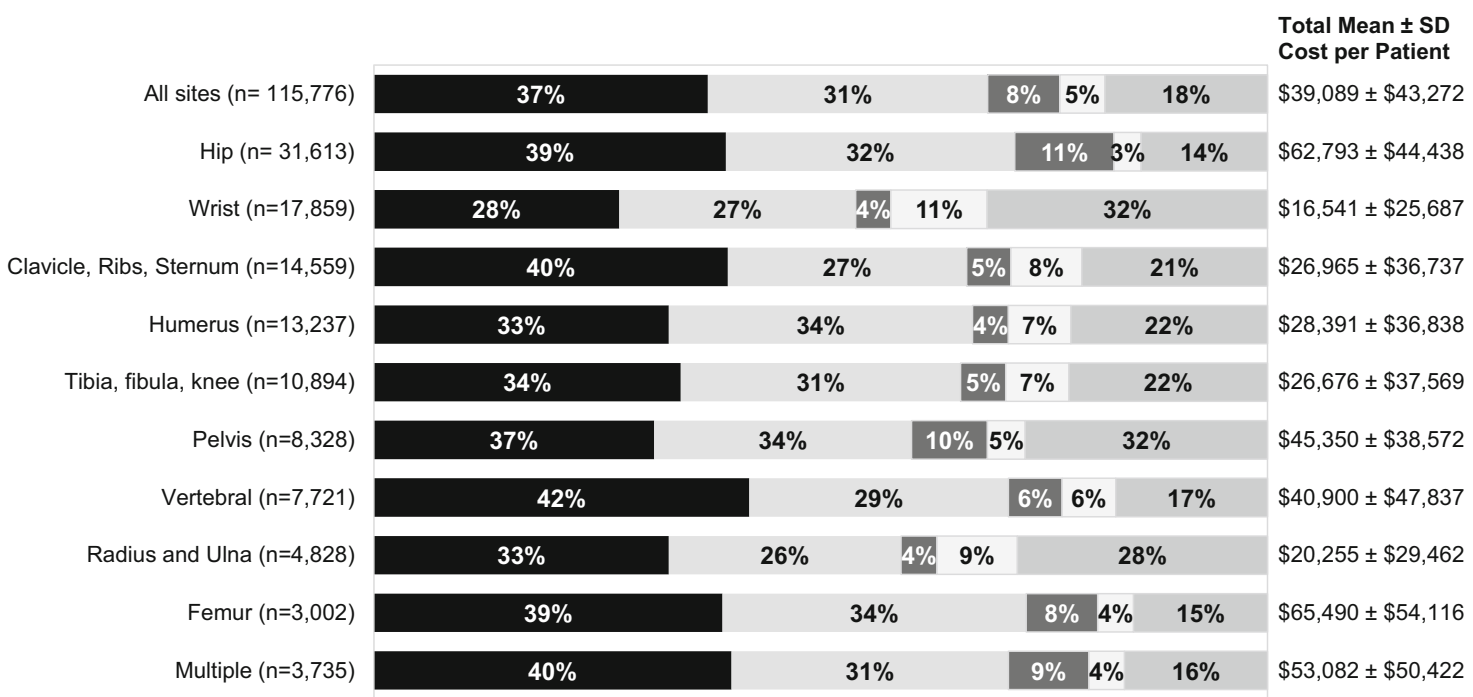

- Hospitalization CCRS, Home care, LTC $\backsim$ Inpatient Rehabilitation $\square$ Prescription Drugs $₫$ Other health care services

Fig. 1 Mean healthcare cost per patient in first year following index fracture, by fracture site, and type of healthcare resource. CCRS, Continuing Care Reporting System; LTC, long-term care; SD, standard deviation. *Other healthcare services include emergency department visits, hospital outpatient clinic visits, physician billings, non-physician billings, and laboratory claims 
Fig. 2 Mean total healthcare costs in the first year after index date for matched fracture and non-facture cohorts. Results presented as mean (SD). CCRS, Continuing Care Reporting System; LTC, long-term care. *Other healthcare services include emergency department visits, hospital outpatient clinic visits, physician billings, non-physician billings, and laboratory claims

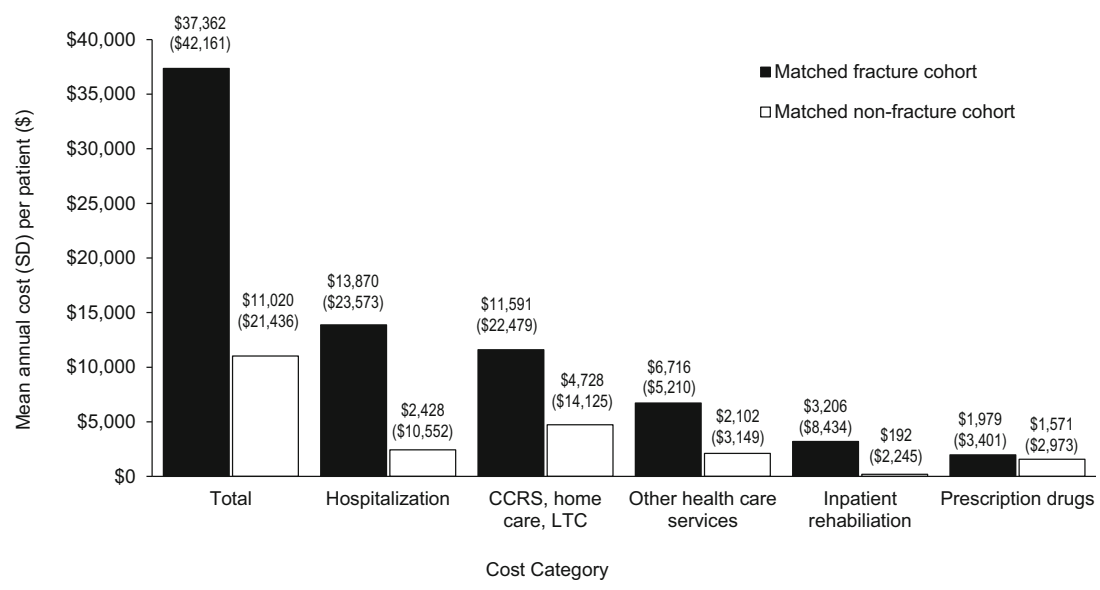

proportion of costs (43\%) for continuing care/home care/LTC. The 1-year excess cost of incident fragility fractures was $\$ 26,341$ per patient, with $43 \%$ of total excess costs attributed to hospitalization and $26 \%$ attributed to continuing care/home care/LTC. Other healthcare services and inpatient rehabilitation represented $18 \%$ and $11 \%$ of the incremental costs, respectively. Prescription drug costs constituted the smallest proportion of total healthcare costs in the fracture cohort and accounted for $2 \%$ of incremental costs. Applying the incremental first-year direct healthcare costs incurred by patients with fragility fractures (i.e., \$26,341) to the average number of incident fragility fractures per year (i.e., 27,489) indicates that the incremental first-year costs associated with fragility fractures represents approximately an annual $\$ 724$ million in Ontario or $\$ 1.9$ billion in Canada. When the total excess cost per patient was varied by $\pm 10 \%$, the incremental costs of fragility fractures extrapolated to Canada ranged from $\$ 1.7$ billion to $\$ 2.1$ billion.

\section{Long-term costs associated with fragility fractures}

Follow-up data was available for 2, 3, 4, and 5 years for $100 \%$ $(n=115,776), 75 \%(n=87,322), 50 \%(n=58,228)$, and $28 \%$ $(n=31,861)$ of patients, respectively (i.e., mean time from index fracture to end of follow-up of 3.26 years). As shown in Fig. 3, considerable healthcare resource utilization costs continued to be incurred in subsequent years following a fragility fracture. The healthcare costs per patient in the second year following a fracture were $\$ 17,139$ and then decreased to $\$ 15,300$ in year $3, \$ 13,613$ in year 4 , and $\$ 12,013$ in year 5

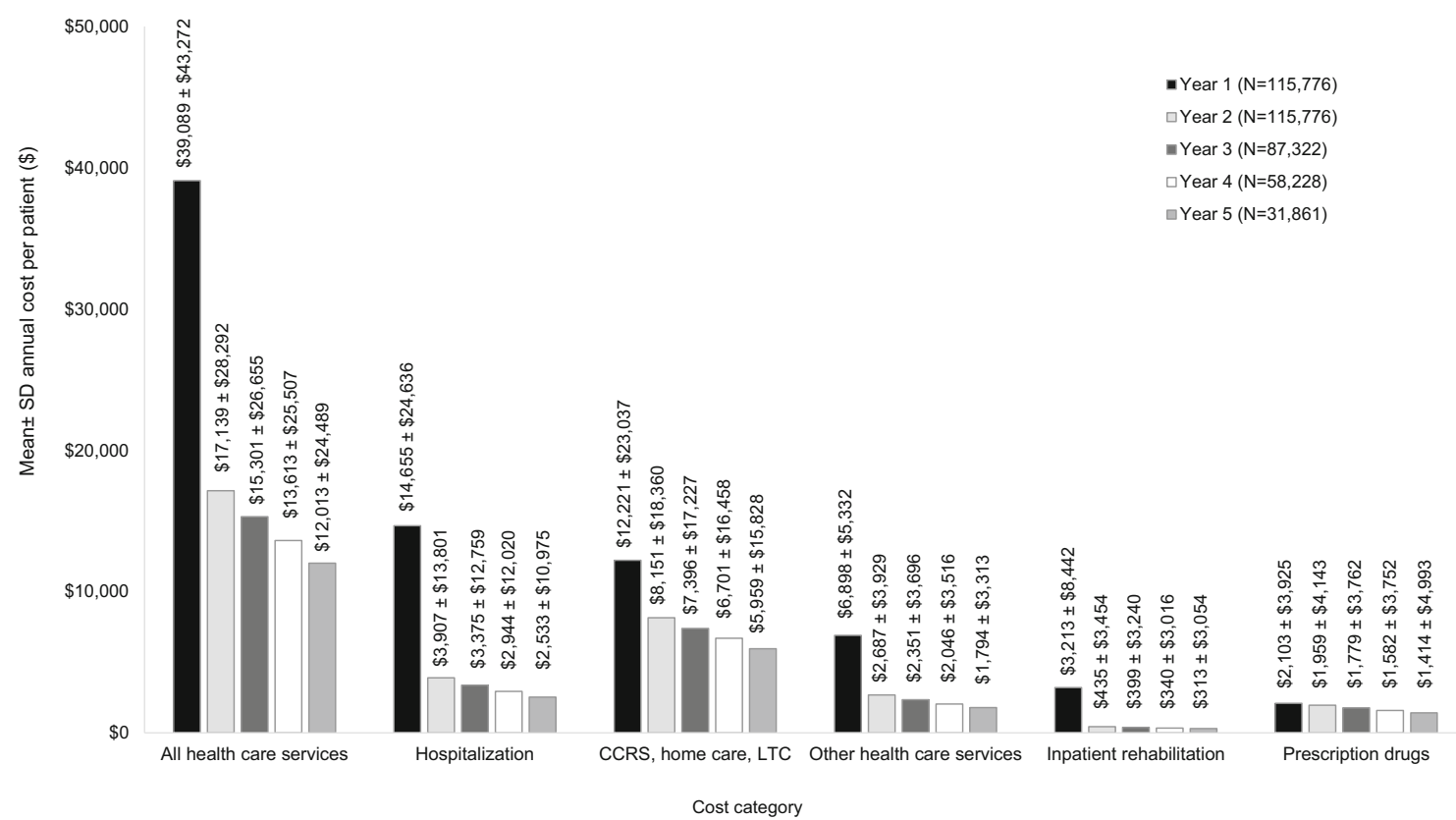

Fig. 3 Mean annual cost per patient up to 5 years after index fracture, by healthcare resource use category. CCRS, Continuing Care Reporting System; LTC, long-term care. *Other healthcare services include emergency department visits, hospital outpatient clinic visits, physician billings, non-physician billings, and laboratory claims 
following the index fracture. Hospitalization was the main driver of costs during the first year following the fracture (37\% of costs) while continuing care/home care/LTC were the main drivers of the costs in years 2-5 following fractures (e.g., $48 \%$ to $50 \%$ of costs). There was less variation in prescription drug costs (\$2103 in year 1 versus $\$ 1414$ in year 5). Online resource Table 3 presents the detailed mean annual cost per patient up to 5 years after index fracture by fracture site and healthcare resource use category.

\section{Discussion}

Using a large population-based incident fracture cohort matched to a non-fracture cohort, our analysis showed that patients with fragility fractures incurred an excess 1-year direct healthcare cost of $\$ 26,341$ across all resource utilization categories compared to those without a fracture. The main cost drivers were hospitalization and continuing care (hospitalbased continuing care, home care, and LTC) across the study period. The first-year incremental healthcare costs of incident fragility fractures for the province of Ontario were estimated at $\$ 724$ million, which translated into an excess $\$ 1.9$ billion annually when extrapolated to Canada. Although the costs varied by fracture site, the costs generally increased with age and number of comorbidities. Costs were highest in the first year following index fracture (mean $\$ 39,089 \pm$ SD $\$ 43,272$ per patient) but remained above $\$ 12,000$ in years $2-5$ following the index fractures.

Comparisons with other Canadian studies are difficult due to differences in time period, databases used, types of healthcare resource utilization captured, and characteristics of patients included in the study cohorts or control groups. Nonetheless, our estimates are higher than those reported before. For example, using a similar methodology and data sources, the 1-year cost in the first year after incident hip fracture in Ontario was estimated at $\$ 52,000-\$ 54,000$ based on 2004-2008 data [15]. In contrast, we reported a first-year cost of $\$ 62,793$ per patient with an incident hip fracture using 2011-2015 data from Ontario. A study from Manitoba estimated the 2-year cost of hip fractures at approximately $\$ 57,000$ and the incremental costs of hip fracture at approximately \$45,000 using data from 2007/2008 [14]; however, this study did not provide the data for year 1 and year 2 separately and the results were based on a small number of hip fractures $(N=751)$. A more recent study from Manitoba [20] used data from 1997 to 2002, but results were expressed in medians making direct comparisons difficult with our study. Although not evaluating the incremental costs of fragility fractures, a Canadian study using national administrative databases estimated the first-year direct medical costs associated with fragility fractures at approximately $\$ 25,000$ per patient (compared to $\$ 39,089$ in our study) [16]. While the study reported that the annual healthcare costs of fragility fractures were $\$ 4.6$ billion (including $\$ 400,000$ for wage loss and mobility devices), these figures were based on incident and prevalent fractures that occurred in 2011 (as opposed to incident fractures in our study). Matched cohort analyses conducted in Europe and the USA have shown the excess costs of hip fracture in the first year [6, 8-10,21], with incremental annual costs as high as $\$ 50,508$ USD per patient in a US Medicare population [7]. Differences in study designs, healthcare systems and clinical practice patterns across countries, and the publication of these data across more than 10 years make it difficult to compare results between studies. However, regardless of the geographic region, there are substantial costs imposed on the healthcare system. While the excess costs were attributed to hospitalization costs in some countries [8, 10], they were largely due to nursing home stays, rehabilitation center stays, and home physical therapy services in others [21]. The excess costs are relevant for healthcare providers to design and implement effective strategies to prevent fractures in high-risk individuals.

Despite the differences in methodology and results across previous Canadian studies and our current analysis [13, 14, 16], the substantial economic burden that fragility fractures place on LTC and rehabilitation services is evident. The current study supports the observation that a large proportion of healthcare costs in the years following a fracture are associated with LTC, which reflects a global trend of an increase in aging populations and an expected increase in the use of LTC [16]. Cost-effective strategies for continuing care services are especially important for older patients since there is a high risk of mortality associated within the first year after experiencing a fragility fracture [22]. Additional surveys are required to monitor the effectiveness of guideline-recommended prevention strategies, especially in older residents.

This study has some limitations which should be noted before drawing any conclusions from the data. The cohort was limited to patients aged $>65$ with public drug coverage (ODB Program) from Ontario and a third of the cohort was older than 85 years which further limits generalizability. The study excluded patients who had experienced a fracture within 5 years prior to the index event but not beyond those 5 years; therefore, the cohort was potentially biased towards an older population. In this incidence-based analysis, patients were followed longitudinally from their index date and approximately one-third of patients had follow-up data available for the full 5-year period. Also, the excess costs of fracture beyond the first year have not been estimated as this was beyond the scope of this study. Another area of future research is to estimate the excess costs in a fixed length cohort (e.g., 5-year survivors). We acknowledge that there is some uncertainty in extrapolating the costs to Canada, as Ontario unit costs may not be representative of costs in other provinces and the population distribution of those 65 years and over varies across 
other provinces (e.g., from $14 \%$ in Alberta to $22 \%$ in New Brunswick; average of $18 \%$ in Canada) [23]. It should be however noted that the population distribution of those 65 years and over is the same in Ontario as Canada overall (i.e., $18 \%$ ), which minimizes the potential bias in extrapolating the Ontario results to Canada based on population data. There is also potentially an underestimation of the number of fractures in the cohort studied, specifically with vertebral fractures, considering that we captured "clinical vertebral fractures" that were identified from hospital/emergency/outpatient clinics and as a result would underestimate those managed in the community (i.e., general practitioner). The cost of prescription drugs only included medications covered under ODB and may underestimate the costs of drugs dispensed at hospitals as these were not captured in the analysis. Direct costs were only included from a public payer perspective and do not include any costs covered through private insurance plans or paid out-of-pocket by the patient (for example non-OHIP funded physiotherapy, occupational therapy). Indirect costs (i.e., productivity loss, absenteeism, presenteeism) were not included and limits the contribution at a societal perspective. The healthcare costs included in this analysis may not be related to the index fragility fracture and could reflect unrelated conditions. No statistical analyses were performed to compare the differences between the matched cohorts and the differences may not be statistically significant. Furthermore, additional analyses would be required to evaluate costs and healthcare resource utilization in other provinces to attain a more holistic understanding of the economic burden of osteoporotic patients in Canada.

Despite these limitations, the strengths of the present study are the large sample size and the matched cohort analysis. The study captured all residents of Ontario older than 65 years with any type of fragility fracture and their associated healthcare resource utilization over a 5 -year period in the largest province in Canada. Patients were matched from the date of index fracture by age, sex, and comorbidities. By conducting a matched analysis with a non-fracture cohort, this mitigated some general limitations observed in previous studies, such as being able to attribute subsequent healthcare resource utilization and costs to the fracture. An added value of the present study is the evaluation of costs up to 5 years following a fragility fracture, as few studies have evaluated the longer term costs beyond the first year or two [20].

\section{Conclusions}

Fragility fractures pose a substantial economic burden to the healthcare system. This is expected to continue to increase with an aging population in Canada. Implementation of improved fracture preventive strategies can result in overall cost savings. Further research is required to determine appropriate management of osteoporotic fracture patients and prevention methods to decrease the burden of fragility fractures on the Canadian healthcare system.

Supplementary Information The online version contains supplementary material available at https://doi.org/10.1007/s00198-021-05877-8.

Acknowledgements This study made use of de-identified data from the ICES Data Repository, managed by the ICES with support from its funders and partners: Canada's Strategy for Patient-Oriented Research (SPOR), the Ontario SPOR Support Unit, the Canadian Institutes of Health Research, and the Government of Ontario.

Funding This study was funded by Amgen Canada Inc.

Data availability The datasets generated during and/or analyzed during the current study are available in the ICES repository upon request.

\section{Declarations}

Ethics approval This retrospective study made use of de-identified aggregate-level data that was obtained from the ICES data repository following patient-level analysis. The study protocol was approved by the Advarra Institutional Review Board.

Consent to participate For this type of study, formal consent is not required.

Consent for publication All authors approved the final version and agree to be accountable for all aspects of the work.

Conflict of interest JET has received consulting fees, research funding, and honoraria from Allergan, AstraZeneca, Amgen, CSL Behring, Janssen, Novo Nordisk, Sage, Assurex/Myriad, Edwards Lifesciences, Pfizer, Roche, Merck, GlaxoSmithKline, Evidera, PCDI, and CADTH. JDA has received consulting fees from Amgen and Eli Lilly; received research funding from Amgen, Radius, Pfizer, and BMS; served on the speakers' bureau for Amgen; and served on boards for the International Osteoporosis Foundation and Ontario Rheumatology Association. JPB has received consulting fees and honoraria from Amgen and Servier; received research funding from Mereo BioPharma, Radius Health, and Servier; and served on speakers' bureau for Amgen. ES has received consulting fees from Amgen. LS is an employee of and owns stock in Amgen. NB is an employee of Amgen. Medical writing support was provided by Amaris Consulting and funded by Amgen Canada Inc.

Disclaimer The opinions, results, and conclusions reported are those of the authors. No endorsement by ICES or any of its funders or partners is intended or should be inferred. Parts of this material are based on data and information compiled and provided by CIHI. However, the analyses, conclusions, opinions, and statements expressed herein are those of the author, and not necessarily those of CIHI.

Open Access This article is licensed under a Creative Commons Attribution-NonCommercial 4.0 International License, which permits any non-commercial use, sharing, adaptation, distribution and reproduction in any medium or format, as long as you give appropriate credit to the original author(s) and the source, provide a link to the Creative Commons licence, and indicate if changes were made. The images or other third party material in this article are included in the 
article's Creative Commons licence, unless indicated otherwise in a credit line to the material. If material is not included in the article's Creative Commons licence and your intended use is not permitted by statutory regulation or exceeds the permitted use, you will need to obtain permission directly from the copyright holder. To view a copy of this licence, visit http://creativecommons.org/licenses/by-nc/4.0/.

\section{References}

1. Johnell O, Kanis JA (2006) An estimate of the worldwide prevalence and disability associated with osteoporotic fractures. Osteoporos Int 17:1726-1733

2. Oden A, McCloskey EV, Kanis JA, Harvey NC, Johansson H (2015) Burden of high fracture probability worldwide: secular increases 2010-2040. Osteoporos Int 26:2243-2248

3. Kanis JA, Oden A, Johnell O, Jonsson B, de Laet C, Dawson A (2001) The burden of osteoporotic fractures: a method for setting intervention thresholds. Osteoporos Int 12:417-427

4. Burge R, Dawson-Hughes B, Solomon DH, Wong JB, King A, Tosteson A (2007) Incidence and economic burden of osteoporosis-related fractures in the United States, 2005-2025. J Bone Miner Res 22:465-475

5. Borgström F, Karlsson L, Ortsäter G, Norton N, Halbout P, Cooper C, Lorentzon M, McCloskey EV, Harvey NC, Javaid MK, Kanis JA, IOF (2020) Fragility fractures in Europe: burden, management and opportunities. Arch Osteoporos 15:59

6. Rousculp MD, Long SR, Wang S, Schoenfeld MJ, Meadows ES (2007) Economic burden of osteoporosis-related fractures in Medicaid. Value Health 10:144-152

7. Adeyemi A, Delhougne G (2019) Incidence and economic burden of intertrochanteric fracture: a medicare claims database analysis. JB JS Open Access 4:e0045

8. Gutierrez L, Roskell N, Castellsague J, Beard S, Rycroft C, Abeysinghe S, Shannon P, Robbins S, Gitlin M (2011) Study of the incremental cost and clinical burden of hip fractures in postmenopausal women in the United Kingdom. J Med Econ 14:99107

9. Gutierrez L, Roskell N, Castellsague J, Beard S, Rycroft C, Abeysinghe S, Shannon P, Gitlin M, Robbins S (2012) Clinical burden and incremental cost of fractures in postmenopausal women in the United Kingdom. Bone 51:324-331

10. De Laet CE, van Hout BA, Burger H, Weel AE, Hofman A, Pols HA (1999) Incremental cost of medical care after hip fracture and first vertebral fracture: the Rotterdam study. Osteoporos Int 10:6672

11. Rabenda V, Manette C, Lemmens R, Mariani AM, Struvay N, Reginster JY (2006) The direct and indirect costs of the chronic management of osteoporosis: a prospective follow-up of 3440 active subjects. Osteoporos Int 17:1346-1352

12. Hiligsmann M, Reginster JY, Tosteson ANA, Bukata SV, Saag KG, Gold DT, Halbout P, Jiwa F, Lewiecki EM, Pinto D, Adachi
JD, al-Daghri N, Bruyère $\mathrm{O}$, Chandran $\mathrm{M}$, Cooper $\mathrm{C}$, Harvey NC, Einhorn TA, Kanis JA, Kendler DL, Messina OD, Rizzoli R, Si L, Silverman S (2019) Recommendations for the conduct of economic evaluations in osteoporosis: outcomes of an experts' consensus meeting organized by the European Society for Clinical and Economic Aspects of Osteoporosis, Osteoarthritis and Musculoskeletal Diseases (ESCEO) and the US branch of the International Osteoporosis Foundation. Osteoporos Int 30:45-57

13. Tarride JE, Hopkins RB, Leslie WD, Morin S, Adachi JD, Papaioannou A, Bessette L, Brown JP, Goeree R (2012) The burden of illness of osteoporosis in Canada. Osteoporos Int 23:25912600

14. Hopkins RB, Tarride JE, Leslie WD, Metge C, Lix LM, Morin S, Finlayson G, Azimaee M, Pullenayegum E, Goeree R, Adachi JD, Papaioannou A, Thabane L (2013) Estimating the excess costs for patients with incident fractures, prevalent fractures, and nonfracture osteoporosis. Osteoporos Int 24:581-593

15. Nikitovic M, Wodchis W, Krahn M, Cadarette S (2013) Direct health-care costs attributed to hip fractures among seniors: a matched cohort study. Osteoporos Int 24:659-669

16. Hopkins RB, Burke N, Von Keyserlingk C, Leslie WD, Morin SN, Adachi JD, Papaioannou A, Bessette L, Brown JP, Pericleous L, Tarride J (2016) The current economic burden of illness of osteoporosis in Canada. Osteoporos Int 27:3023-3032

17. ICES (2020) ICES data. https://www.ices.on.ca/Data-and-Privacy/ ICES-data. Accessed 20 Aug 2020

18. Avis D, Davis B, Steele JM (1988) Probabilistic analysis of a greedy heuristic for euclidean matching. Probab Eng Inf Sci 2: $143-156$

19. Wodchis WP, Bushmeneva K, Nikitovic M, McKillop I (2013) Guidelines on person-level costing using administrative databases in Ontario. Working Paper Series. Vol 1. Toronto: Health System Performance Research Network.

20. Leslie WD, Lix LM, Finlayson GS, Metge CJ, Morin SN, Majumdar SR (2013) Direct healthcare costs for 5 years postfracture in Canada: a long-term population-based assessment. Osteoporos Int 24:1697-1705

21. Haentjens P, Autier P, Barette M, Boonen S, Belgian Hip Fracture Study Group (2001) The economic cost of hip fractures among elderly women. A one-year, prospective, observational cohort study with matched-pair analysis. Belgian Hip Fracture Study Group. J Bone Joint Surg Am 83:493-500

22. Brown JP, Adachi JD, Schemitsch E, Tarride JE, Brown V, Bell A, Reiner M, Oliveira T, Motsepe-Ditshego P, Burke N, Slatkovska L (2021) Mortality in older adults following a fragility fracture: realworld retrospective matched-cohort study in Ontario. BMC Musculoskelet Disord 22:105

23. Statistics Canada. Table 17-10-0005-01 Population estimates on July 1st, by age and sex. https://www150.statcan.gc.ca/t1/tbl1/en/ tv.action?pid=1710000501 Accessed 17 Dec 2020

Publisher's note Springer Nature remains neutral with regard to jurisdictional claims in published maps and institutional affiliations. 